\title{
La faim comme origine de la parole
}

Jérôme THELOT

\section{(2) OpenEdition}

Journals

Édition électronique

URL : http://journals.openedition.org/transtexts/590

DOI : $10.4000 /$ transtexts.590

ISSN : 2105-2549

Éditeur

Gregory B. Lee

Référence électronique

Jérôme THELOT, «La faim comme origine de la parole », Transtext(e)s Transcultures 跨文本跨文化 [En ligne], 10 | 2015, mis en ligne le 14 juin 2016, consulté le 19 avril 2019. URL : http://

journals.openedition.org/transtexts/590; DOI : 10.4000/transtexts.590

Ce document a été généré automatiquement le 19 avril 2019.

(c) Tous droits réservés 


\title{
La faim comme origine de la parole
}

\author{
Jérôme THELOT
}

Une dernière visite fut rendue aux nids d'albatros sur le petit plateau onduleux, parmi les touffes d'herbe, les plaques de neige et les mares gelées. [...]. Très souvent, un des parents veillait auprès du nid. Cela ne nous réjouissait guère d'attaquer ces oiseaux ; mais la faim ne connaît pas de loi, et puis ils étaient d'un goût si agréable et contribuaient si bien à nous rendre des forces que chaque fois que nous venions à en tuer un, notre remords s'en trouvait singulièrement diminué. ${ }^{1}$

1 La faim ne connaît pas de loi - Shackleton, revenu de son exploration du pôle Sud, est de ceux qui l'ont dit mieux que d'autres - parce qu'elle est la loi de toutes les lois. La faim précède chronologiquement et phénoménologiquement toutes les lois possibles, puisqu'elle est leur condition originaire. Elle conditionne toute loi et n'en connaît aucune, étant l'archi-loi qui ne connaît qu'elle même et ne veut rien savoir d'autre. La faim ne veut rien savoir en particulier de ce que sait, quant à lui, le remords de Shackleton et de ses hommes, quand, explorateurs affamés, ils tuent les jeunes albatros sur la banquise, pour ne pas mourir de faim. À proprement parler, elle ne peut rien savoir d'autre, ne pouvant d'abord que se savoir elle-même. Et encore faut-il dire que ce savoir d'elle-même auquel elle est vouée n'est en vérité pas un savoir: car la faim ne se sait pas, elle s'éprouve; elle n'est pas un savoir, mais un affect. C'est comme affect originaire qu'elle est l'archi-loi de toute loi et la condition de tout savoir possible. En l'occurrence, la faim est aussi la condition du savoir anthropologique et philosophique, lequel peut bien la perdre de vue, la méconnaître ou la dissimuler, mais ne peut pas faire qu'il ne s'origine en elle. La faim est non pas le commencement de la philosophie mais la plus ancienne passivité conditionnant tout commencement intellectuel, ou culturel, ou symbolique possible. De sorte que l'étonnement auquel la philosophie a pu identifier son commencement vient d'un oubli, l'oubli de cet affect en la profondeur duquel toute pensée et toute symbolisation sont précédées. Étonnant oubli! Si le philosophe qui s'étonne d'être, et qui s'étonne de l'Être, ne sait pas que cet étonnement est conditionné par l'affect originaire de la faim, c'est en vertu d'une situation qui alimente (c'est le cas de le dire) son savoir et sa capacité de savoir, situation qu'on nomme la satiété. L'oubli de la faim est la satiété, laquelle est la situation du philosophe, de l'anthropologue, de 
l'interprète des symboles et des rites, dont le savoir méconnaît la faim en laquelle pourtant son savoir s'origine.

La recherche ici entreprise - sur la symbolique des arts culinaires - ne peut avancer que si les chercheurs ont le ventre plein, assez plein du moins pour oublier la faim, pour que la pensée librement avance. C'est un enseignement important de l'exploration du pôle par Shackelton sonné au travail intellectuel: de même que l'exploration du pôle vire en chasse aux albatros, la faim s'y substituant à la découverte escomptée, et s'imposant comme la véritable aventure aussi ancienne qu'inattendue, de même le travail intellectuel rencontrera bientôt, quand les chercheurs sentiront au ventre une fringale qui les empêchera de réfléchir, sa condition de possibilité - car il faudra bientôt manger. Exploration et recherche se reconnaitront subordonnées à l'heure de leur renoncement respectif : quand il faudra que la science abdique, quand il faudra poser la plume pour un repas, parce qu'il faut manger pour vivre. La faim s'exécute ainsi au revers de la recherche - exploration ou colloque - comme la sensation d'un il faut, à laquelle elle devra céder. D'où la nécessité maintenant de réfléchir un peu sur cet il faut antérieur au repas, sur cet affect primordial de la faim non symbolique, non verbale, non culturelle, antérieure à tout langage et hétérogène et à toute institution.

\section{L'affect originaire}

Rimbaud, Une saison en enfer :

Jadis, si je me souviens bien, ma vie était un festin où s'ouvraient tous les cœurs, où tous les vins coulaient. ${ }^{2}$

4 Cette phrase, la première d'Une saison en enfer, l'une des plus belles qui soient parmi les phrases de Rimbaud, précipite d'un coup l'unité fondamentale du moi, de «ma » vie, de cette « vie » mienne, et du « festin » qu'elle était. Que ma vie fût un festin, cela veut dire que la relation entre moi et ma vie, entre ma vie et moi, était une relation d'immanence et d'intériorité réciproque. Moi-même intérieur à ma vie et ma vie intérieure à moimême, cela se produisait selon la pure immédiateté d'une faim heureuse - un «festin » -, laquelle est le bonheur premier. Jadis, ma faim était comblée, j’étais la faim comblée, c'était le bonheur et c'était la vie, cette vie mienne, identique au bonheur de vivre.

Inversement l'enfer, comme dit Rimbaud, c'est la faim quand elle n'est pas comblée, ou plus comblée, ou donc le malheur. De sorte que l'entreprise du poète passant « une saison en enfer " se décrit comme suit : "J'ai songé à rechercher la clef du festin ancien, où je reprendrais peut-être appétit ». Le poète recherche de ce qui est perdu quand la faim n'est pas comblée et quand l'appétit s'absente, une recherche plus exactement de l'« appétit » quand même le festin perdu se retrouverait. Si non seulement l'enfer est la faim, mais si en outre la clef cherchée par le condamné est celle qui lui rouvrirait l'appétit, alors ce damné vit la contradiction la plus grande. C'est à la faim qu'il est condamné puisqu'il a perdu le festin ancien, mais c'est à l'« appétit » qu'il consacre sa recherche : sa condamnation, c'est que son besoin est clivé, que son appétit manque à sa faim, qu'il a faim de la faim qu'il n'a plus.

6 Jadis, sa vie était la sienne, et réciproquement son soi était sa vie, et c'était cela le "festin », cette coïncidence originaire du soi et de la vie, cette épiphanie réciproque du soi comme vie et de la vie comme soi. Quel «festin », le festin de la vie! La vie est le bonheur avec lequel la subjectivité se donne à elle-même comme comblement de sa faim. 
La vie est la donation réciproque de la faim et de son comblement, est la venue à soi de la subjectivité en tant que cette donation. Si je me souviens bien, ma vie était cette faim qu'elle ne peut pas ne pas être, cette faim dans laquelle le vivre s'accomplit, inépuisable, " où tous les vins coulaient ", et surabondante, " un festin ", qui donnant satisfaction à ma faim lui donnait conjointement de s'augmenter elle-même. La vie comme festin est l'accroissement réciproque du rassasiement et de la faim : plus j'étais rassasié plus j'avais faim, plus j'avais faim plus j'étais rassasié.

7 Voilà pourquoi, a contrario, la définition de l'enfer que Rimbaud donne est rigoureuse, rigoureusement phénoménologique : l'enfer est la faim clivée, celle non seulement qui n'est pas comblée mais qui manque d'appétit, la condamnation à vivre hors du « festin » de la vie. L'enfer est un clivage de la subjectivité. Car que « Ma vie fût un festin », cela veut dire: d'abord il n'est de faim que mienne, et il n'est de moi que d'abord faim. Autrement dit, la faim est l'affect originaire dans la révélation duquel la subjectivité parvient à soi, le mode premier de la venue à soi de la subjectivité. L'archi-donation en laquelle se produit la subjectivité est l'apparaître à soi de la faim. La subjectivité n'est pas une substance parce que la faim n'en est pas une, elle est un apparaître affectif. « Jadis, si je me souviens bien " : la faim est antérieure à toute substance, à toute conscience, à toute identité comme à toute liberté, a fortiori elle est antérieure à toute culture, à toute élaboration symbolique, et à proprement parler elle n'est rien d'historique, car me donnant à moi-même elle m'a toujours précédé, plus vieille que moi qui la souffre depuis toujours qu'elle m'advient. Elle n'est en effet pas, au sens où moi je finis par être, car elle donne : elle me donne à moi en se donnant avant toute autre phénomène et en particulier avant tout langage et avant toute mémoire. Si je me souviens bien, jadis, dans un passé plus lointain que tout passé, avant les jours dont je me souviens, avant la mémoire avec laquelle mes souvenirs remontent aux jours mémorables, si je me souviens bien, donc, autrement que selon la mémoire, autrement que selon les représentations, si je me souviens d'un immémorial qui est l'émotion essentielle à laquelle je suis dû - ma vie était un festin. Et cet archi-passé dans lequel j'éprouve le festin de ma faim, cet immémorial irreprésentable dans l'émotion duquel, toujours, elle m'arrive, c'est son mode propre d'apparition. Elle m'a toujours déjà atteint anhistoriquement d'une atteinte me livrant à moi-même : j'ai faim donc je suis.

\section{L'll me faut}

Homère, L'Iliade :

Car même Niobé aux beaux cheveux a songé à manger,

Elle à qui douze enfants dans sa maison périrent,

Six filles et six fils à la fleur de leur âge.

Eux, Apollon les tua avec son arc d'argent

Dans sa colère contre Niobé ; elles, Artémis qui aime les flèches.

C'est qu'elle s'était égalée à Lèto aux belles joues,

Disant "elle a deux enfants ; moi, j'en ai enfanté beaucoup".

Et ces deux, quoiqu'ils ne fussent que deux, les ont fait tous mourir.

Eux neuf jours furent gisants dans la mort; nul ne vint

Les enterrer. Les gens étaient devenus des pierres par le vouloir de Zeus.

Et eux le dixième jour furent ensevelis par les dieux du ciel.

Mais elle a songé à manger, quand elle fut fatiguée des larmes. ${ }^{3}$ 
Que Niobé qui a perdu ses douze enfants vienne, ayant faim, à manger, et ainsi à oublier ses fils, ses filles, et ses larmes, c'est la pire misère selon Homère, qui en éprouve autant d'amertume que de sollicitude. La faim est la pire misère car elle prive de tout, même de pouvoir endurer la misère. Et même de Niobé la faim a congédié la douleur. Cette pire misère n'a rien à voir avec la guerre sévissant alentour. Ce n'est pas que les enfants de Niobé furent tués en temps de guerre, qui dernièrement importe. Ce ne sont pas les horreurs de la guerre qui inspirent ici la dernière amertume, c'est cette servitude pire que la guerre et si accaparante que s'y oublient même ses horreurs, c'est ce pire malheur d'oublier le plus intime malheur. L'amertume d'Homère a pour motif, non la violence ni la mort, mais l'indifférence à la violence et l'infidélité aux morts, et que même Niobé ne soit pas digne de sa souffrance. La souffrance humaine est sans grandeur, si la force de la faim est plus envahissante que l'empire de la guerre, si la nécessité de manger est plus contraignante que le deuil des enfants. La question de savoir si vraiment la faim est ignoble autant qu'elle est humiliante, si vraiment Niobé s'avilit d'y perdre sa douleur, ou si ce n'est pas, au contraire, le comble de l'humain qui lui arrive avec cette faim - qui la soustrait pourtant à ses devoirs de mère -, cette question est morale comme celle de la guerre. Mais la méditation de Homère, ici, n'est pas d'abord morale ni politique. Suivre Homère, c'est approcher la nécessité antérieure à toute histoire et conditionnant celle-ci, extérieure à toute politique et où celle-ci a son fondement, cette nécessité absolue, transcendantale, qui est l'il faut manger radicalement subjectif. La pire misère est l'originaire, celle selon Homère de la persévérance du vivant dans sa vie, du vouloir-vivre de sa persévérance. Niobé apparaît à Homère et s'apparaît à elle-même dans sa soumission irrémissible à cette « force » qui se veut elle-même, et qui la veut, que Spinoza appellera le conatus essendi, et Schopenhauer le vouloir-vivre. Mais cette soumission est son assujettissement: elle s'y trouve comme sujet. La "force», à la fois antérieure et immanente à Niobé, à laquelle elle ne peut que se soumettre, et qui, se la soumettant, la donne à elle-même, est l'archi-subjectivité. L'il faut manger, cette pire misère suscitant l'amertume et la sollicitude du poète, cette nécessité plus ancienne que la morale et que la politique, cette force d'avant même le langage, est l'il faut de Niobé, où celle-ci vient à elle-même. Autrement dit, l'il faut est nécessairement d'un sujet: c'est un il «me» faut. Non seulement la faim ne s'impose que pour autant qu'elle arrive à quelqu'un, mais elle le fait du dedans de son essence, selon une arrivée qui lui est totalement immanente, non pas du dehors de ce qu'il serait sans elle, comme s'il pouvait ne pas lui être assujetti, mais comme l'épreuve qui le produit et lui est identique. Il faut manger pour vivre, signifie : il me faut manger, c'est ainsi que je vis; ou identiquement : il me faut vivre, c'est ainsi que j'ai faim.

\title{
L'll me faut parler
}

Rimbaud, Fêtes de la faim :

\author{
Ma faim, Anne, Anne, \\ Fuis sur ton âne. \\ Si j'ai du goût ce n'est guères \\ Que pour la terre et les pierres. \\ Dinn! dinn! dinn! dinn ! je pais l'air, \\ Le roc, les terres, le fer.
}


11 Si la faim est l'affect non seulement originaire, mais aussi subjectivant, alors il convient sans doute de la décrire comme l'événement même de la vérité. Or c'est ce que fait Rimbaud dans ce petit poème.

« Fuis sur ton âne ». Qu'il faille que la faim fuie, c'est d'abord conforme à ce qu'a dégagé la description précédente. Car fuir signifie disparaître, et il faut en effet que la faim disparaisse dans son retournement en satiété. C'est même précisément là son mode d'apparition, cet il faut disparaître dans un repas où elle doit fuir : l'apparaître de la faim est ce vouloir intérieur, pathétiquement éprouvé, de sa disparition. Mais il y a aussi autre chose, et c'est l'apport du poème qu'il force ici la pensée à s'y reprendre : car fuir signifie également échapper (à un danger, à un poursuivant), décamper pour se garder sauf, fuis sur ton âne signifie donc: fuis ce repas qui te menace, évite cette satiété où tu disparaîtrais, garde-toi de manger. De sorte que celui, dans ce poème, qui parle à sa faim comme d'autres parlent à leur âme: "Ma faim, Anne, Anne ", et qui lui parlant de cette façon se parle, ce poète qui se rejoint dans cette faim qu'il éprouve, mais qui, cependant, dans ce rapport verbalisé à soi, dans cette adresse réflexive, s'établit aussi comme une conscience de sa faim, une conscience autre que sa faim dont elle est conscience, ce poète par conséquent divisé d'avec soi, clivé entre d'une part sa faim et d'autre part sa conscience, d'une part son affect et d'autre part sa verbalisation, réclame de sa faim d'un seul coup deux choses contraires: fuis dans le repas où disparaître, et fuis le repas pour apparaître. Or cette double injonction en une seule formule ambiguë est la verbalisation du mouvement dont toute faim est faite, du mouvement de sa disparition dans le repas et de sa réapparition dès la satiété, de la fuite du besoin dans le manger et de la fuite de la satiété dans le besoin; si bien que ce que l'injonction contradictoire de la conscience à la faim comprend de celle-ci, c'est cet accomplissement de la faim comme telle, son mode propre de manifestation, cette alternance sienne de la souffrance et du bonheur. Fuis sur ton âne, veut dire : disparais-apparais, mange-ne-mange-pas. De sorte que la leçon de ces vers, c'est que la conscience tient à la faim: elle en recueille la phénoménalité, elle en verbalise synthétiquement le mouvement alternatif, et elle en réclame la persévérance. La conscience tient à la faim non seulement comme à ce dont elle est conscience, mais comme à ce qui la conditionne dans son ambiguité propre. Cette thèse donc s'impose : la faim est l'origine de la conscience, l'affect fonde la verbalisation, l'épreuve affective de soi est la condition transcendantale du rapport symbolique à soi.

13 Il y a donc une poiétique de la faim, en un sens fondamental. La faim essentiellement fait parler, elle est cela qui fait parler. L'expérience capitale d'Arthur Rimbaud n'est pas en profondeur d'avoir connu la faim et d'avoir connu la soif (vraisemblablement dès la prime enfance par des frustrations oubliées, ensuite quand sa mère l'aura châtié par des privations de repas, puis dans ses fugues où sa fatigue creusait sa marche, et à Londres faute d'argent, et toujours jusqu'à la fin de par le monde), mais d'avoir reconnu que la faim est la vérité. Autrement dit, Rimbaud a su - et a su dire - que la faim est l'archirelation à soi de la subjectivité en laquelle celle-ci se manifeste, d'abord selon l'affect corporel, ensuite selon la conscience verbale. La faim est la vérité, cela signifie : c'est sur le fond de la faim et surdéterminé par elle que ce qui advient peut advenir, du corps ou de la conscience ; et le langage lui-même, et l'histoire et la symbolicité ont dans cette épreuve originaire leur condition de possibilité la plus essentielle. C'est parce que la faim est ce qui fait parler qu'on appelle "nourrisson » le nouveau né, qui se fait connaître par ses cris de faim : si le nourrisson est l'être parlant, c'est comme parlant de sa faim. Ainsi, l'obéissance à la nécessité s'accomplit poiétiquement, et ne peut pas ne pas le faire. 
L'épreuve de l'affect s'exécute comme expérience de la conscience. La venue à soi de la subjectivité dans l'il me faut devient représentation de soi comme affamé. Le pathos tourne en signes, en allégories, et l'intériorité de la faim s'extériorise en langage. L'il me faut veut dire; et il veut dire ceci : il me faut dire.

\section{Le mangeable et l'immangeable. Du monde}

Dante, La Divine Comédie, «L'Enfer », Chant trente-troisième :

De son repas féroce il retira la bouche, Ce pécheur, et l'ayant essuyée aux cheveux

Du crâne qu'il avait par derrière entamé,

Il commença. ${ }^{4}$

Dans son trou du neuvième cercle de l'Enfer, Ugolino, pour parler, doit soulever de son repas sa bouche, cette bouche formidable par laquelle Dante est si fasciné qu'il ouvre avec elle le premier vers du Chant : « La bocca sollevo dal fiero pasto ».

Car comme on sait, ce repas qu'Ugolino suspend consiste à ronger, «au point où le cerveau se relie à la nuque ", son bourreau Ruggieri, parce que celui-ci, sur terre, lui avait infligé un supplice épouvantable dont Dante consigne le récit. Ugolino réduit à sa bouche, le récit de ses malheurs procède d'une oralité saturée : parole prise au repas, expression avérant sa continuité avec la manducation, extériorisation verbale d'une consommation charnelle. La bouche ne cesse pas d'être première dans la parole comme dans le repas, dans l'évocation du supplice comme dans la dévoration du bourreau. Donc ce supplice, on le connaît, la bouche d'Ugolino le raconte.

Enfermé avec ses enfants dans la Tour de la Faim, Ugolino a rêvé que Ruggieri, avec ses chiens, chassait un loup et ses louveteaux, il s'est mordu les poings, il a entendu ses enfants lui proposer qu'il les mange, il les a vus mourir de faim l'un après l'autre, il est devenu aveugle, il les a pleurés, et il les a touchés dans l'ombre de la Tour : «Et puis ce que la douleur ne put, la faim le put » («Poscia, piu che 'l dolor, poté 'l digiuno »). Or ce vers, le plus ambigu et l'un des plus fameux de La Divine Comédie, dit à la fois et indécidablement qu'Ugolino mourut de faim (d'une faim plus cruelle que sa douleur) et qu'il mangea ses enfants (d'une faim que même sa douleur n'aura pu retenir), sans qu'une hypothèse jamais puisse l'emporter sur l'autre, ni une certitude s'ensuivre. Borges a parlé avec justesse de cette ambiguïté, du point de vue de la narration et de la littérature, en faisant valoir qu'elle donne au texte sa profondeur et son pouvoir sur l'imagination du lecteur ${ }^{5}$. Mais c'est sur un autre plan qu'il faut trouver la grandeur du texte. Le poème de Dante est grand parce qu'il montre droitement la bouche - la bocca - et que toute ambiguité se ressource en celle-ci, la bouche ouverte d'abord dans la Tour de la faim, puis dans le trou du neuvième cercle de l'enfer, ensuite dans le récit de la victime retournée en bourreau, et enfin dans le poème. C'est par la bouche que tout vient et revient : le récit d'Ugolino et le récit de Dante, le supplicié se mordant les poings et mangeant une cervelle, la victime dévorée par sa faim et dévorant son bourreau, la parole des enfants qui meurent de faim et celle des damnés dont la faim ne meurt jamais. Dans la bouche qui parle et qui mange, qui parle comme elle mange, qui se tait quand elle mange et mange quand elle se tait, dans cette bouche non par hasard mais par essence le premier mot du poème, ce que Dante entend et fait entendre c'est la jonction originaire de la parole et de la manducation, plus exactement leur continuité phénoménologique à partir de la même faim, où l'une comme l'autre trouve sa nécessité. 

même de la nécessité : il lui faut parler, il ne peut pas ne pas le faire, et c'est donc de l'il me faut qu'il lui faut parler. Robert Antelme, revenu des camps de la mort, a dit cette intrication, dans la bouche, de la faim et des mots. Il est permis de citer son livre après ceux de Rimbaud et de Dante :

Quand je n'ai plus rien eu dans la bouche, le vide a été insupportable. Encore, encore ; le mot a été fait pour la langue et le palais; encore une bouchée, encore une bouchée, il ne fallait pas que ça s'arrête, la machine à broyer, à sentir, à lécher était en marche. La bouche n'avait jamais éprouvé comme à ce moment-là qu'elle était une chose qui ne pouvait pas être comblée, que rien ne pouvait lui servir une fois pour toutes, qu'il lui en faudrait toujours. ${ }^{\circ}$ que celui dont sont morts tous les morts de faim de l'histoire universelle: c'est le tourment de la chair privée de s'oublier, à laquelle on a retiré le monde qui la nourrirait, autrement dit le supplice, exactement, de la vérité.

Ugolino a peut-être mangé ses enfants, peut-être pas. Mais ce qu'enseignerait une certitude à ce propos serait moins utile que ce qu'enseigne cette incertitude. Car celle-ci donne à penser la possibilité qu'il les ait mangés, et cette possibilité elle-même est la définition $\mathrm{du}$ «monde » tel qu'il vient à la faim. Le monde en effet est ce qui vient à la faim, le monde vient si nécessairement à la faim que celle-ci le trouve même dans la Tour où les prisonniers sont enfermés, elle le trouve parce qu'il le lui faut, en dépit de la prison et de l'impossibilité de s'évader, en dépit des interdits et des remords, en dépit de tout, elle le trouve comme cette nécessité de s'oublier, de disparaître sans mourir, dans n'importe quoi qui la délivre de soi. Ugolino, sa bouche, doit trouver le monde même dans sa Tour, le monde comme cette possibilité de manger (même ses propres enfants). Robert Antelme, de même, observant parmi les détenus l'un de ceux qui ont pu se restaurer, dont le corps a donc des rondeurs, note aussi sobrement que le ferait Dante : « On pourrait sans doute manger un homme tel que celui-ci. » Mais Ugolino, en revanche, ne peut pas manger les pierres de sa prison, comme Robert Antelme ne peut pas manger l'air de son camp : «Les dents mâchent l'air et la salive. Le corps est vide. Rien que de l'air dans la bouche, dans le ventre, dans les jambes et dans les bras qui se vident. » Les pierres et l'air sont immangeables, comme le fer chez Rimbaud, comme les poches du manteau chez Knut Hamsun, comme les chaussures de Charlot dans le film de Chaplin.

Or cette différence entre le mangeable et l'immangeable, entre l'impossibilité de manger les poches de son manteau et la possibilité de manger ses propres enfants, cette différence est la condition sous laquelle le monde se produit, est la possibilité originaire du monde, et du coup sa définition phénoménologique rigoureuse. La différence entre mangeable et immangeable est la différence première à partir de laquelle les autres différences - historiques, langagières, culturelles - se constituent. Et d'abord elle détermine la distinction entre l'intérieur et l'extérieur, entre le corps propre et le dehors. Car le corps propre, même quand la faim crée à tout prix du mangeable, lui-même demeure immangeable, - c'est d'ailleurs la raison pour laquelle il est possible de torturer par la faim. Si Ugolino a mangé même ses enfants, ensuite il n'aura pourtant pas pu se manger lui-même, et c'est cette impossibilité qui fit sa condamnation. On sait que Diogène, s'adonnant à la masturbation sur la place publique, expliquait à raison : « Ah ! si seulement en se frottant aussi le ventre, on pouvait calmer sa faim. ${ }^{7}$ " De même, Robert Antelme : «Je mâche, je mâche, mais soi, ça ne se mâche pas. Je suis celui qui mâche, mais ce qui se mâche, ce qui se mange, où cela existe-t-il ? ${ }^{8}$ »

Transtext(e)s Transcultures 跨文本跨文化, 10 | 0000 
Les différences culturelles, celles qu'élaborent les sociétés au gré de leurs histoires, celles dont l'art culinaire est la mise en œuvre, distribuent le mangeable et l'immangeable selon des intérêts réels et imaginaires, selon des interdits et des mythes, selon des conditions environnementales et des projets sociaux, des anticipations et des craintes, des héritages et des situations, des représentations et des tabous, de sorte que se distingueront aussi, dans chaque société ou micro-société, les gras et les maigres, ceux qui ont droit à ce qui se mange et ceux qui n'y ont pas droit, ceux qui nomment les différences et ceux qui les subissent. Gras et maigres, repus et affamés, gloutons et gourmets, boulimiques et anorexiques, mangeants et mangés, jeûneurs et consommateurs, etc., apparaissent dans le monde avec les différentes pratiques alimentaires, sur le fonds de cette différence initiale entre mangeable et immangeable, elle-même ramifiée par les différences sensibles qu'éprouveront les individus, le doux et l'amer, le gluant et le croustillant, l'excitant et l'émollient, le digeste et l'indigeste, etc. Quand donc le Tentateur - ce sera mon dernier exemple -, dans l'Évangile de Matthieu, demande au Christ qui a jeûné au désert pendant quarante jours, de prouver qu'il est Dieu en métamorphosant les pierres du désert en pains, il lui suggère d'abolir cette différence fondamentale, instauratrice, entre mangeable et immangeable, il tente de ruiner la poïétique de la faim : il veut défaire, avec la faim, la possibilité même du monde ${ }^{9}$. Le refus que lui oppose le Christ vient de la conscience de celui-ci, de la valeur absolue de la faim. Car on le sait, l'homme ne se nourrit pas que de pain.

\section{NOTES}

1. Sir E. Shackleton, L'Odyssée de l' 'Endurance', Texte français de M.-L. Landel, Éditions Phébus, 1988, p. 197-198.

2. Rimbaud, Une saison en enfer, CEuvres complètes, Gallimard, Pléiade, 1972, p. 93.

3. S. Weil, L'Iliade ou le poème de la force, CEuvres, Gallimard, Quarto, 1999, p. 534. (Il s'agit de la traduction du chant XXIV, v. 602-613.)

4. Dante, La Divine Comédie, Traduction par H. Longnon, Garnier, 1966, p. 163.

5. Jorge Luis Borges, « Le faux problème d'Ugolin », Neuf essais sur Dante, Traduction de Fr. Rosset, Gallimard, « Arcades », 1987.

6. Robert Antelme, L'Espèce humaine, Édition revue et corrigée, Gallimard, Tel, 1993, p. 112-113.

7. Diogène Laërce, Vies et doctrines des philosophes illustres, Traduction de M.-O. Goulet-Cazé, Le Livre de poche, 1999, p. 736 (voir aussi p. 722).

8. L'Espèce humaine, op. cit., p. 144.

9. Matthieu, IV, 1-3. 


\section{RÉSUMÉS}

La faim est l'affect originaire en lequel la subjectivité arrive à soi ; elle est l'archi-fonds indubitable antérieur à la réflexion de la conscience, la loi antérieure à toute loi, l'anhistorique épreuve de soi par laquelle est fondée l'histoire elle-même. Ainsi convient-il de la décrire sous le titre d'Il me faut, comme la condition transcendantale en laquelle trouvent leur possibilité le langage et le politique, l'intentionnalité et le monde. Ce sont les poètes et les romanciers qui donnent accès à cette pensée de la faim comme origine de toute parole. 